\title{
Ergodic Properties of Equilibrium States
}

\author{
Joseph Slawny
}

Laboratory for Transport Theory and Mathematical Physics, Virginia Polytechnic Institute and State University Blacksburg, VA 24061, USA

\begin{abstract}
Possible ergodic properties of Gibbs states are discussed by constructing a number of examples. In particular existence of Gibbs states which are mixing but not extremal is shown.
\end{abstract}

Any invariant state $\varrho$ which is an extremal Gibbs state, [2], [6], [8], is mixing [6], i.e. for any $f, g \in L^{2}(\varrho)$

$$
\varrho\left(f \cdot \tau_{a} g\right) \rightarrow \varrho(f) \varrho(g) \quad \text { as } \quad|a| \rightarrow \infty .
$$

In this note we discuss the question to what extent the converse holds, i.e. if any mixing Gibbs state is extremal. In particular, we construct an example of a mixing non-extremal Gibbs state.

More precisely, we consider a hierarchy of ergodic properties : ergodicity, weak mixing, $n$-fold mixing $(n>2)$, extremality in the set of all Gibbs states, each property being stronger than the preceding one. By considering suitable ferromagnetic finite range interactions we obtain equilibrium states which are

- ergodic but not weakly mixing;

- weakly mixing but not mixing;

- mixing (i.e., 2-fold mixing) but not 3-fold mixing, and thus not extremal Gibbs states.

Construction of ergodic but not weakly mixing states is trivial: average over translations of any periodic not $\mathbb{Z}^{v}$-invariant extremal Gibbs state yields here an example. One can obtain such examples already in two dimensions. The other examples are more complicated and are constructed in dimension three or higher. At the end of this note we discuss a result of Ledrappier [7] which stimulated working out of the examples below.

We adopt the following definitions [4], [1]. Let $\mathscr{X}$ be a compact $\mathbb{Z}^{v}$-space (i.e., $\mathbb{Z}^{v}$ acts on $\mathscr{X}$ by homeomorphisms). We denote by $f \mapsto \tau_{a} f, a \in \mathbb{Z}^{v}$, the induced action of $\mathbb{Z}^{v}$ on $C(\mathscr{X})$ and by $E^{I}$ the family of all $\mathbb{Z}^{v}$-invariant states of $C(\mathscr{X})$. For 
$a=\left(a^{1}, \ldots, a^{v}\right) \in \mathbb{Z}^{v}, n>0$, finite $\Lambda \subset \mathbb{Z}^{v}$, we let

$$
\begin{aligned}
|a| & =\max \left\{\left|a^{i}\right|: i=1, \ldots, v\right\}, \quad \Lambda(n)=\left\{a \in \mathbb{Z}^{v}:|a|<n\right\} \\
|\Lambda| & =\operatorname{card} \Lambda .
\end{aligned}
$$

A set $J \subset \mathbb{Z}^{v}$ is of zero density if $|J \cap \Lambda(n)| /|\Lambda(n)| \rightarrow 0$ as $n \rightarrow \infty$. A state $\varrho \in E^{I}$ is ergodic, respectively: weakly mixing, mixing, if for any $f, g \in C(\mathscr{X})$

$$
\lim _{n \rightarrow \infty} \frac{1}{|\Lambda(n)|} \sum_{x \in \Lambda(n)} \varrho\left(f \cdot \tau_{x} g\right)=\varrho(f) \varrho(g)
$$

respectively:

$$
\begin{aligned}
\lim _{n \rightarrow \infty} & \frac{1}{|\Lambda(n)|} \sum_{x \in \Lambda(n)}\left|\varrho\left(f \cdot \tau_{x} g\right)-\varrho(f) \varrho(g)\right|=0, \\
& \lim _{|x| \rightarrow \infty} \varrho\left(f \cdot \tau_{x} g\right)=\varrho(f) \varrho(g)
\end{aligned}
$$

$\varrho \in E^{I}$ is $n$-fold mixing if for any $f_{1}, \ldots, f_{n} \in C(\mathscr{X})$

$$
\lim _{\substack{\left|x_{i}-x_{j}\right| \rightarrow \infty \\ i \neq j}} \varrho\left(\tau_{x_{1}} f_{1} \cdot \tau_{x_{2}} f_{2} \ldots \tau_{x_{n}} f_{n}\right)=\varrho\left(f_{1}\right) \cdot \ldots \cdot \varrho\left(f_{n}\right) .
$$

Weak mixing is equivalent to existence for any $f, g \in C(\mathscr{X})$ of a set $J$ of zero density such that

$$
\lim _{|x| \rightarrow \infty, x \notin J} \varrho\left(f \cdot \tau_{x} g\right)=\varrho(f) \varrho(g) .
$$

We note that to check any of the above properties it is enough to verify it for a family of functions, linear combinations of which are dense in $C(\mathscr{X})$.

Invariant extremal Gibbs states are $n$-fold mixing for each $n$, [6]. Thus an equilibrium state which is mixing but not 3 -fold mixing is not an extremal Gibbs state.

The present paper is, in a sense, a continuation of [9], and we adopt the notation of that reference. In particular $\mathscr{X}=\{-1,1\}^{\mathbb{L}}, \mathbb{L}=\mathbb{Z}^{v}$, is the configuration space of a spin $1 / 2$ system with the natural action of $\mathbb{Z}^{v} ; \sigma_{A}$, where $A$ is a finite subset of $\mathbb{L}$, is the usual product of spin variables and the (translation invariant) Hamiltonian is

$$
-\sum_{B \in \mathscr{B}} \sigma_{B}
$$

We will consider only finite range interactions: in each case there is a finite subfamily $\mathscr{B}_{0} \subset \mathscr{B}$ such that each element of $\mathscr{B}$ is a translate of $\mathscr{B}_{0} . \varrho^{+}$is the translation invariant extremal Gibbs state corresponding to inverse temperature $\beta$ obtained with " +1 - boundary conditions at infinity", and $\varrho^{0}$ is the thermodynamic limit of $\varrho_{\Lambda}^{0}$ as $\Lambda \rightarrow \mathbb{L}$.

$$
\varrho_{\Lambda}^{0}(X)=\frac{\exp \beta \sum_{B \subset A, B \in \mathscr{B}} \sigma_{B}(X)}{\sum_{Y} \exp \beta \sum_{B \subset A, B \in \mathscr{B}} \sigma_{B}(Y)} .
$$


$\varrho^{0}$ is translation invariant but not extremal in general. Most of our examples are obtained by taking $\varrho^{0}$ for suitable $\mathscr{B}$ and large $\beta$.

For two subsets $C, D$ of $\mathbb{L}$ let $C+D$ be their symmetric difference

$$
C+D=(C \backslash D) \cup(D \backslash C) .
$$

Then $\mathscr{P}_{f}(\mathbb{L})$, the family of all finite subsets of $\mathbb{L}$, with the + operation is an (abelian) group; let $\overline{\mathscr{B}}$ be the subgroup of $\mathscr{P}_{f}\left(\mathbb{Z}^{v}\right)$ generated by $\mathscr{B}$.

For any interaction of the form (1)

$$
\begin{aligned}
& \varrho^{0}\left(\sigma_{A}\right)=0 \quad \text { if } \quad A \notin \overline{\mathscr{B}} \\
& \varrho^{0}\left(\sigma_{A}\right)=\varrho^{+}\left(\sigma_{A}\right) \text { if } \quad A \in \overline{\mathscr{B}}
\end{aligned}
$$

and $\beta$ is large enough [10], and for all models we will consider, [5] allows us to check that

$$
\varrho^{+}\left(\sigma_{A}\right) \neq 0 \quad \forall A \in \mathscr{P}_{f}(\mathbb{L}), \text { for large } \beta \text {. }
$$

Let $\left(M_{n}\right), n=1,2,3, \ldots$, be the following property of $\overline{\mathscr{B}}\left(A_{1}, \ldots, A_{n}\right.$ are elements of $\left.\mathscr{P}_{f}(\mathbb{L})\right):\left(M_{1}\right)$ for any $A_{1}, A_{2} \notin \overline{\mathscr{B}}$ there exists a set $J \subset \mathbb{Z}^{v}$ of density zero such that $A_{1}+\tau_{x}\left(A_{2}\right) \notin \overline{\mathscr{B}}$ if $x \notin J$.

$$
\begin{gathered}
\left(M_{n}, n \geqq 2\right) \quad \text { for any } A_{1}, \ldots, A_{n} \notin \overline{\mathscr{B}}, \\
\tau_{x_{1}}\left(A_{1}\right)+\tau_{x_{2}}\left(A_{2}\right)+\ldots+\tau_{x_{n}}\left(A_{n}\right) \notin \overline{\mathscr{B}}
\end{gathered}
$$

if all $\left|x_{i}-x_{j}\right|, i \neq j$, are large enough. Assuming (2)-(4) we will now show that for $n \geqq 2$, if $\varrho^{0}$ is $n$-mixing then $\left(M_{n}\right)$ holds (in fact $\varrho^{0}$ is $n$-mixing if $\left(M_{k}\right)$ holds for $k \leqq n$ ). For if $A_{1} \ldots A_{n} \notin \overline{\mathscr{B}}$ then for $n$-mixing $\varrho^{0}$

$$
\varrho^{0}\left(\prod_{i=1}^{n} \tau_{x_{i}}\left(\sigma_{A_{i}}\right)\right) \rightarrow \prod_{i=1}^{n} \varrho^{0}\left(\sigma_{A_{i}}\right)
$$

as $\min _{i \neq j}\left|x_{i}-x_{j}\right| \rightarrow \infty$, and $\prod_{i=1}^{n} \varrho^{0}\left(\sigma_{A_{i}}\right)=0$ by (2). On the other hand if $\left(x_{k, i}\right) i=1, \ldots, n$, $k=1,2, \ldots$ are such that $\left|x_{k, i}-x_{k, j}\right| \geqq k, i \neq j$, and $\sum_{i=1}^{n} \tau_{x_{k, i}}\left(A_{i}\right) \in \overline{\mathscr{B}}$ then

$$
\begin{aligned}
\varrho^{0}\left(\prod_{i=1}^{n} \tau_{x_{k, i}}\left(\sigma_{A_{i}}\right)\right) & =\varrho^{0}\left(\begin{array}{c}
\sigma_{\sum_{i=1}^{n}} \tau_{x_{k, i}}\left(A_{i}\right) \\
\sum_{i=1}
\end{array}\right)=\varrho^{+}\left(\prod_{i=1}^{n} \tau_{x_{k, i}}\left(\sigma_{A_{i}}\right)\right) \rightarrow \prod_{i=1}^{n} \varrho^{+}\left(\sigma_{A_{i}}\right), \\
& =\varrho^{+}\left(\sigma_{x_{i}} \tau_{i}\right)
\end{aligned}
$$

as $k \rightarrow \infty$, which is non-zero by (4); here the first and the third equality follow from $\sigma_{C} \cdot \sigma_{D}=\sigma_{C+D}(\forall C, D)$ and second from (3) and $\sum_{i=1}^{n} \tau_{x_{k, i}}\left(A_{i}\right) \in \overline{\mathscr{B}}$. Similarly one shows that for weakly mixing $\varrho^{0},\left(M_{1}\right)$ holds.

On the other hand, $\left(M_{1}\right)$ implies that $\varrho^{0}$ is weakly mixing; since linear combinations of $\left\{\sigma_{A}: A \in \mathscr{P}_{f}(L)\right\}$ are dense in $C(\mathscr{X})$ it is enough to show that for any 
$A_{1}, A_{2}$ there exists a set $J \subset \mathbb{Z}^{v}$ of density zero such that

$$
\varrho^{0}\left(\sigma_{A_{1}} \cdot \tau_{x}\left(\sigma_{A_{2}}\right)\right) \underset{|x| \rightarrow \infty, x \notin J}{\longrightarrow} \varrho^{0}\left(\sigma_{A_{1}}\right) \varrho^{0}\left(\sigma_{A_{2}}\right) .
$$

If both $A_{1}$ and $A_{2}$ are in $\overline{\mathscr{B}}$ then by (3) and the fact that $\varrho^{+}$is mixing one can take $J=\emptyset$; if $A_{1} \in \overline{\mathscr{B}}, A_{2} \notin \overline{\mathscr{B}}$ then $A_{1}+\tau_{x}\left(A_{2}\right) \notin \overline{\mathscr{B}}$ and by $(2) \varrho^{0}\left(\sigma_{A_{1}} \cdot \tau_{x}\left(\sigma_{A_{2}}\right)\right)=0$ and $\varrho^{0}\left(\sigma_{A_{1}}\right) \varrho^{0}\left(\sigma_{A_{2}}\right)=0$ and therefore $\left(^{*}\right)$ holds with empty $J$. If $A_{1}, A_{2} \notin \overline{\mathscr{B}}$ let $J$ be as in $\left(M_{1}\right)$. Then

$$
\varrho^{0}\left(\sigma_{A_{1}} \cdot \tau_{x}\left(\sigma_{A_{2}}\right)\right)=\varrho^{0}\left(\sigma_{A_{1}+\tau_{x}\left(A_{2}\right)}\right)
$$

is zero for $x \notin J$ (by (2)) and (*) holds again.

In exactly the same way one shows that $\left(M_{2}\right)$ implies that $\varrho^{0}$ is mixing. Thus we see that $\varrho^{0}$ is weakly mixing but not mixing if $\left(M_{1}\right)$ holds but $\left(M_{2}\right)$ does not hold, and that $\varrho^{0}$ is mixing but not 3-fold mixing if $\left(M_{2}\right)$ holds but $\left(M_{3}\right)$ does not hold. Since (4) depends on $\overline{\mathscr{B}}$ only, and not on $\mathscr{B}$, corresponding ergodic properties of $\varrho^{0}$ at low temperatures depend only on $\overline{\mathscr{B}}$ too.

Example 1. (layered Ising Model). $\left(M_{1}\right)$ holds but $\left(M_{2}\right)$ does not. $\mathbb{L}=\mathbb{Z}^{3}$, $\mathscr{B}_{0}=\left\{B_{1}, B_{2}\right\}$,

$$
B_{1}=\left\{0, e_{1}\right\}, \quad B_{2}=\left\{0, e_{2}\right\} .^{1}
$$

The same $\overline{\mathscr{B}}$, and therefore the same ergodic properties of $\varrho^{0}$ at low temperatures, are obtained if we enlarge $\mathscr{B}_{0}$ by

$$
B_{3}=\left\{0, e_{1}, e_{3}, e_{1}+e_{3}\right\}
$$

which yields a "connected" model.

Here $A \in \overline{\mathscr{B}}$ iff each plane perpendicular to $e_{3}$ intersects $A$ at an even number of points. Thus if $A_{1}, A_{2} \notin \overline{\mathscr{B}}$ then $A_{1}+\tau_{x} A_{2} \notin \overline{\mathscr{B}}$ for all $x$ with large enough $x_{3}$, which proves $\left(M_{1}\right)$. To see that $\left(M_{2}\right)$ does not hold it is enough to take $A_{1}=A_{2}=\{0\}$ and observe that $A_{1}+\tau_{n e_{1}} A_{2} \in \mathscr{\mathscr { B }}$ for all $n$. (4) holds here since $\varrho^{+}$for this model is a product of corresponding states of two-dimensional Ising models for which (4) holds. Thus we obtain a Gibbs state which is weakly mixing but not mixing. Another example is provided by the model of [9, Sect. 4].

We conjecture that as soon as $\mathscr{P}_{f}(\mathbb{L}) / \overline{\mathscr{B}}$ is infinite and $(4)$ holds, $\varrho^{0}$ is weakly mixing.

Example 2. $\left(M_{2}\right)$ holds, $\left(M_{3}\right)$ does not; the proofs are much more complicated than in Example 1. $\mathbb{L}=\mathbb{Z}^{3}, \mathscr{B}_{0}=\left\{B_{1}, B_{2}\right\}$,

$$
B_{1}=\left\{0, e_{1}, e_{2}\right\}, \quad B_{2}=\left\{e_{1}, e_{3}, 2 e_{3}\right\} .
$$

To prove $\left(M_{2}\right)$ we first notice that it is enough to check it for $A_{2}=A_{1}$. For suppose for any $n$ there exists $x \in \mathbb{Z}^{3}$ such that $|x|>n$ and $a_{1}+\tau_{x} A_{2} \in \overline{\mathscr{B}}$. Then for any $n$ there exist $x_{1}, x_{2} \in \mathbb{Z}^{3}$ such that $\left|x_{1}-x_{2}\right|>n$ and $A_{1}+\tau_{x_{1}} A_{2} \in \overline{\mathscr{B}}, A_{1}+\tau_{x_{2}} A_{2} \in \overline{\mathscr{B}}$. But then

$$
\tau_{x_{2}}\left(A_{2}+\tau_{x_{1}-x_{2}} A_{2}\right)=\tau_{x_{1}} A_{1}+\tau_{x_{2}} A_{2}=\left(A_{1}+\tau_{x_{1}} A_{2}\right)+\left(A_{2}+\tau_{x_{2}} A_{2}\right) \in \overline{\mathscr{B}}
$$

and therefore $A_{2}+\tau_{x_{1}-x_{2}}\left(A_{2}\right) \in \overline{\mathscr{B}}$. Now we reformulate the problem in the algebraic language of [5] and use the algebra to solve it.

$1 e_{1}, \ldots, e_{v}$ is the canonical basis of $\mathbb{Z}^{v}$ 
In the notation of $\left[5\right.$, Sect. 3], writing $X=\left\{e_{1}\right\}, Y=\left\{e_{2}\right\}, Z=\left\{e_{3}\right\}$, $x=(\alpha, \beta, \gamma) \in \mathbb{Z}^{3}$, we have

$$
A+\tau_{x}(A)=\left(+X^{\alpha} Y^{\beta} Z^{\gamma}\right) \cdot A,
$$

where the multiplication on the right hand side is understood in the sense of $\mathbb{F}_{2}\left[\mathbb{Z}^{3}\right]\left(\equiv \mathscr{P}_{f}\left(\mathbb{Z}^{3}\right)\right)$. Now $A+\tau_{x}(A) \in \overline{\mathscr{B}}$ means that there are $P, Q \in \mathbb{F}_{2}\left[\mathbb{Z}^{3}\right]$ such that

$$
\left(1+X^{\alpha} Y^{\beta} Z^{\gamma}\right) \cdot A=P \cdot B_{1}+P \cdot B_{2} .
$$

Multiplying both sides here by a suitable monomial $X^{\alpha^{\prime}} Y^{\beta^{\prime}} Z^{\gamma^{\prime}}$, and assuming $A \subset \mathbb{Z}_{+}^{3}$ we see that there exist non-negative integers $\lambda, \mu, \eta, \varrho, \sigma, \tau,(\lambda, \mu, \eta) \neq(\varrho, \sigma, \tau)$, and $P, Q \in \mathbb{F}_{2}[X, Y, Z]$ such that

$$
\left(X^{\lambda} Y^{\mu} Z^{\eta}+X^{\varrho} Y^{\sigma} Z^{\tau}\right) \cdot A=P \cdot B_{1}+Q \cdot B_{2} .
$$

Let $R=X^{\lambda} Y^{\mu} Z^{v}+X^{\varrho} Y^{\sigma} Z^{\tau}$; then (5) is equivalent to the fact that $R \cdot A$ is in the ideal $\mathscr{I}$ of $F_{2}[X, Y, Z]$ generated by $B_{1}, B_{2}$. We will now show that if $A \notin \mathscr{I}$ then the preceding statement implies that $R \in \mathscr{I}$ which in turn will be shown to lead to a contradiction.

Let $\Phi$ be a homomorphism $\mathbb{F}_{2}[X, Y, Z] \rightarrow \mathbb{F}_{2}[Z]$ such that

$$
\Phi(X)=Z+Z^{2}, \quad \Phi(Y)=1+Z+Z^{2}, \quad \Phi(Z)=Z ;
$$

$\Phi\left(\mathbb{F}_{2}[X, Y, Z]\right)=\mathbb{F}_{2}[Z] \quad$ and $\quad$ since $\quad B_{1}=1+X+Y, \quad B_{2}=X+Z+Z^{2}$, $\Phi\left(B_{1}\right)=\Phi\left(B_{2}\right)=0$, i.e., $\mathscr{I} \subset \operatorname{ker}(\Phi)$. In fact $\mathscr{I}=\operatorname{ker}(\Phi)$. For, as is easy to see, each element of $\mathbb{F}_{2}[X, Y, Z]$ is congruent $\bmod \overline{\mathscr{B}}$ to an element of $\mathbb{F}_{2}[Z]$ and $\operatorname{ker}(\Phi)$ has trivial intersection with the later.

Now, if $R \cdot A \in \mathscr{I}$ then $\Phi(R) \cdot \Phi(A)=\Phi(R \cdot A)=0$ and since $\mathbb{F}_{2}[Z]$ has no zero divisors it follows that either $\Phi(R)=0$ or $\Phi(A)=0$. But $\Phi(A)=0$ would contradict $A \in \overline{\mathscr{B}}$. Hence $\Phi(R)=0$, which is the same as

$$
\left(Z+Z^{2}\right)^{\lambda}\left(1+Z+Z^{2}\right)^{\mu} Z^{\eta}=\left(Z+Z^{2}\right)^{\varrho}\left(1+Z+Z^{2}\right)^{\sigma} Z^{\tau}
$$

or

$$
(1+Z)^{\lambda}\left(1+Z+Z^{2}\right)^{\mu} Z^{\lambda+\eta}=(1+Z)^{\varrho}\left(1+Z+Z^{2}\right)^{\sigma} Z^{\varrho+\tau} .
$$

Since the polynomials $1+Z, 1+Z+Z^{2}, Z$ are prime in $\mathbb{F}_{2}[Z]$, the last identity implies that $\lambda=\varrho, \mu=\sigma, \lambda+\eta=\varrho+\tau$, in contradiction with $(\lambda, \mu, \eta) \neq(\varrho, \sigma, \tau)$.

The above argument shows also that if $m \neq n$ then $1+Z^{m}$ and $1+Z^{n}$ are not congruent $\bmod \overline{\mathscr{B}}$, that therefore $\mathscr{P}_{f}(\mathbb{L}) / \overline{\mathscr{B}}$ is infinite, and that in particular one point subsets are not in $\overline{\mathscr{B}}$. Since, obviously,

$$
\mathrm{g} \cdot \mathrm{c} \cdot \mathrm{d} \mathscr{B}=\mathrm{g} \cdot \mathrm{c} \cdot \mathrm{d}\left(1+X+Y, X+Z+Z^{2}\right)=1,
$$

by [5], (4) holds. Thus to show that, for large $\beta, \varrho^{0}$ is not 3 -fold mixing, it is enough to exhibit sequences $\left(a_{n}\right)^{\infty},\left(b_{n}\right)^{\infty},\left(c_{n}\right)^{\infty}$, of points of $\mathbb{L}$ such that $\left\{a_{n}, b_{n}, c_{n}\right\} \in \overline{\mathscr{B}}$ and

$$
\min \left\{\left|a_{n}-b_{n}\right|,\left|a_{n}-c_{n}\right|,\left|b_{n}-c_{n}\right|\right\} \rightarrow \infty \text { as } n \rightarrow \infty .
$$

To this end, since $B_{1}^{2 n} \in \overline{\mathscr{B}}$ and

$$
B_{1}^{2^{n}}=\left\{2^{n} \cdot a: a B_{1}\right\}=\left\{0,2^{n} e_{1}, 2^{n} e_{2}\right\}
$$


[5, Sect. 3.9], define $a_{n}=0, b_{n}=2^{n} e_{1}, c_{n}=2^{n} e_{2}$. Then the above conditions are satisfied proving that $\left(M_{3}\right)$ does not hold.

Remark. As is not hard to see the above proof of $\left(\mathrm{M}_{2}\right)$ depends on two properties of $\overline{\mathscr{B}}$ : on $\overline{\mathscr{B}}$ being a prime ideal of $\mathbb{F}_{2}\left[\mathbb{Z}^{3}\right]$ (we used the homomorphism $\Phi$ to prove it) and on $\overline{\mathscr{B}}$ not containing two-point sets. The first condition is the harder to satisfy and to check since it requires the elements of $B$ to be of an irregular shape.

We note that as soon as $\overline{\mathscr{B}}_{f} \neq \mathscr{P}_{f}(\mathbb{L}),\left(M_{m}\right)$ is not satisfied for $m$ large enough : if $A$ is any element of $\overline{\mathscr{B}}, A \neq \emptyset, n=\operatorname{Card} A$, then $\left(M_{n}\right)$ does not hold. The proof repeats the argument preceding the remark:

$$
A^{2^{m}}=\left\{2^{m} a: a \in A\right\} \in \overline{\mathscr{B}}
$$

consists of $n$ points with separation increasing to infinity as $m \rightarrow \infty$.

We now put our construction in a different perspective. Considering the natural group structure on $\mathscr{X}, \sigma_{A}$ are characters of $\mathscr{X}$ and

$$
\mathscr{S}=\left\{X \in \mathscr{X}: \sigma_{B}(X)=1\right\}
$$

is a compact $\mathbb{Z}^{v}$-invariant subgroup of $\mathscr{X} . \mathscr{S}$ acts on the set of Gibbs states [9], $G \in \mathscr{S}: \varrho \mapsto \varrho_{G}$, and for a probability measure $\mu$ on $\mathscr{S}$ let

$$
\varrho_{\mu}=\int_{\mathscr{S}} \mu(d G) \varrho_{G}^{+},
$$

then

$$
\varrho_{\mu}\left(\sigma_{A}\right)=\mu\left(\sigma_{A}\right) \varrho^{+}\left(\sigma_{A}\right),
$$

where $\mu\left(\sigma_{A}\right)$ is the integral with respect to $\mu$ of the restriction of the character $\sigma_{A}$ to the subgroup $\mathscr{S}$ of $\mathscr{X}$. Suppose now $\varrho^{+}\left(\sigma_{A}\right) \neq 0, \forall A \in \mathscr{P}_{f}(\mathbb{L})$. Then since $\varrho^{+}$is $n$-fold mixing for each $n, \varrho_{\mu}$ is $n$-fold mixing iff $\mu$ is. If $\lambda$ is the Haar measure of $\mathscr{S}$ then $\lambda\left(\sigma_{A}\right)=1$ if $\sigma_{A} \uparrow \mathscr{S}=1$ and $\lambda\left(\sigma_{A}\right)=0$ otherwise, i.e.

$$
\begin{array}{rll}
\lambda\left(\sigma_{A}\right)=1 & \text { if } & A \in \overline{\mathscr{B}} \\
0 & \text { if } & A \notin \overline{\mathscr{B}} .
\end{array}
$$

Therefore for large enough $\beta, \varrho_{\lambda}$ coincides with $\varrho^{0}$, and thus ergodic properties of $\varrho^{0}$ are related to ergodic properties of the Haar measure of $\mathscr{S}$ under the action of $\mathbb{Z}^{v}$ by automorphisms of $\mathscr{S}$. In particular, $\left(M_{1}\right)$ is equivalent to weak mixing, and $\left(M_{n}\right), n \geqq 2$, to $n$-fold mixing of the Haar measure.

In case of an action of $\mathbb{Z}$ by automorphisms of a compact abelian group, ergodicity is equivalent to mixing [1], [4]. In case of a $\mathbb{Z}^{v}$-action, $v>1$, ergodicity is still equivalent to weak mixing but as Example 1 shows it does not imply 2 -fold mixing anymore.

While I have been aware for some time, [11], of the above construction of Gibbs states with various mixing properties, the present examples have been worked out in Spring 1979 after hearing from A. Katok about Ledrappier's example (cf. below). The state constructed in [7] can also be considered as a Gibbs state, in the sense of [8, Chap. 1]. The interaction would then be zero but due to the fact that the space is small (in particular, the pressure is zero) it has the somewhat pathological property, when compared with the usual systems of statistical mechanics, that every state is a Gibbs state. 
The example of Ledrappier [7] fits into our framework as follows. His space $X$ is the group $\mathscr{S} \subset\{1,1\} \mathbb{Z}^{2}$ of $\mathscr{B}$ consisting of translates of

$$
B=\left\{0,2 e_{1}, 2 e_{2}, 2 e_{1}+2 e_{2}, e_{1}+e_{2}\right\} .
$$

According to the Remark, to obtain mixing here it is enough to show that $\overline{\mathscr{B}}$ is a prime ideal of $\mathbb{F}_{2}\left[\mathbb{Z}^{2}\right]$ and that it does not contain a two point set. $\overline{\mathscr{B}}$ is prime since $\overline{\mathscr{B}}$ is a principal ideal generated by the prime element

$$
B=1+X \cdot Y+X^{2}+Y^{2}+X^{2} Y^{2},
$$

and $\overline{\mathscr{B}}$ does not contain a two-point set since it obviously does not contain sets with less than four elements and the argument of the remark shows that the system is not 5-fold mixing. The same argument shows that the measure of the $\mathscr{S}$ defined by translates of

$$
B=\left\{1, e_{1}, e_{2}\right\}
$$

is mixing but not 3 -fold mixing. And more generally, on $\mathbb{Z}^{\nu}$, if $\mathscr{B}$ consists of translates of a $B$ which is prime in $\mathbb{F}_{2}\left[\mathbb{Z}^{v}\right]$ and which is not "one-dimensional", the group $\mathscr{S}$ with the Haar measure and the natural action of $\mathbb{Z}^{v}$ provides a system which is mixing but not $|B|$-mixing.

Our examples have to be more complicated than the above since in two dimensions ferromagnetic Gibbs systems have finite number of phases at low temperatures and no principal ideals are allowed for $\mathscr{B},[5]$. Finally, we note that our argument with $B^{2^{n}}$ shows that $\varrho^{+}$is the only state of the form $\varrho_{\mu}$ which is $n$-fold mixing for any $n$. Thus existence of Gibbs states which are $n$-fold mixing for each $n$ but not extremal is an open question.

Acknowledgement. I wish to thank A. Katok for a stimulating discussion. The hospitality of the Institute for Advanced Study where part of this work has been done is gratefully acknowledged. At VPI \& SU this work has been supported in part by U.S. Department of Energy Grant DE-AS05-80ER10711.

\section{References}

1. Brown, J.R.: Ergodic Theory and Topological Dynamics. New York: Academic Press 1976

2. Dobrushin, R.L.: Funct. Anal. Appl. 2, 292-301 (1968)

3. Gruber, C., Hintermann, A., Merlini, D.: Group Analysis of Classical Lattice Systems. In: Lecture Notes in Physics, Vol. 60. Berlin, Heidelberg, New York: Springer 1976

4. Halmos, P.R.: Lectures in Ergodic Theory. Publ. Math. Soc. Japan, No. 3, Tokyo 1956

5. Holsztynski, W., Slawny, J.: Commun. Math. Phys. 66, 147 (1979)

6. Lanford, O.E., Ruelle, E.: Commun. Math. Phys. 13, 194 (1969)

7. Ledrappier, F.: C.R. Acad. Sci. Paris Ser. A, 287, 561 (1978)

8. Ruelle, D.: Thermodynamic Formalism. Reading, Mass: Addison-Wesley 1978

9. Slawny, J.: Commun. Math. Phys. 35, 297 (1974)

10. Slawny, J.: Commun. Math. Phys. 46, 75 (1976)

11. Slawny, J.: Asterisque 40, 181 (1976)

Communicated by E. Lieb 
